\title{
Adaptive Control using Residual Mode Filters Applied to Wind Turbines
}

\author{
Susan A. Frost ${ }^{1}$ \\ NASA Ames Research Center, Moffett Field, CA 94035 \\ and \\ Mark J. Balas ${ }^{2}$ \\ University of Wyoming, Laramie, WY, 82071
}

\begin{abstract}
Many dynamic systems containing a large number of modes can benefit from adaptive control techniques, which are well suited to applications that have unknown parameters and poorly known operating conditions. In this paper, we focus on a model reference direct adaptive control approach that has been extended to handle adaptive rejection of persistent disturbances. We extend this adaptive control theory to accommodate problematic modal subsystems of a plant that inhibit the adaptive controller by causing the open-loop plant to be non-minimum phase. We will augment the adaptive controller using a Residual Mode Filter (RMF) to compensate for problematic modal subsystems, thereby allowing the system to satisfy the requirements for the adaptive controller to have guaranteed convergence and bounded gains. We apply these theoretical results to design an adaptive collective pitch controller for a high-fidelity simulation of a utility-scale, variable-speed wind turbine that has minimum phase zeros.
\end{abstract}

\section{Introduction}

M any plants, including wind turbines and rotor craft, have modal subsystems that interfere with the ability of an adaptive controller to perform in a stable manner. In this paper, we extend our model reference adaptive control theory [1]-[4] to accommodate modal subsystems of a plant that inhibit the adaptive controller. In particular, residual modes that have non-minimum phase zeros can inhibit the controller by interfereing with the almost strict positive real condition. This theory applies to linear time invariant systems that can be diagonalized or placed into modal form. Linear flexible structures are good examples of appropriate applications of this theory.

The controller modification will use the idea of Residual Mode Filters (RMF) introduced for fixed gain controllers in [5]. In this paper, the RMF will be used to eliminate the effect of modes that prevent the almost strict positive realness of the overall system. These modes have non-minimum phase zeros.

\section{Direct Model Reference Adaptive Control with Rejection of Persistent Disturbances}

In this paper, we focus on the direct adaptive control (DAC) approach developed in [1]-[2] and extended in [3]-[4] to handle adaptive rejection of persistent disturbances. The plant used in this section of the paper will be modeled by the linear, time-invariant, finite-dimensional system:

$$
\left\{\begin{array}{l}
\dot{x}_{p}=A x_{p}+B u_{p}+\Gamma u_{D} \\
y_{p}=C x_{p} ; x_{p}(0)=x_{0}
\end{array}\right.
$$

\footnotetext{
${ }^{1}$ Research Engineer, Intelligent Systems Division, M/S 269-3, AIAA Member.

${ }^{2}$ Professor and Department Head, Electrical and Computer Engineering, AIAA Fellow.
} 
where the plant state, $x_{p}(t)$, is an $\mathrm{N}_{\mathrm{p}}$-dimensional vector, the control input vector, $u_{p}(t)$, is M-dimensional, and the sensor output vector, $y_{p}(t)$, is P-dimensional. The disturbance input vector, $u_{D}(t)$, is $\mathrm{M}_{\mathrm{D}}$-dimensional and will be thought to come from the Disturbance Generator:

$$
\left\{\begin{array}{l}
u_{D}=\Theta z_{D} \\
\dot{z}_{D}=F z_{D} ; z_{D}(0)=z_{0}
\end{array}\right.
$$

where the disturbance state, $z_{D}(t)$, is $\mathrm{N}_{\mathrm{D}}$-dimensional. All matrices in Eqs. (1)-(2) have the appropriate compatible dimensions. Such descriptions of persistent disturbances were first used in [6] to describe signals of known form but unknown amplitude. Equation (2) can be rewritten as in [3] in a form that is not a dynamical system, which is sometimes easier to use:

$$
\left\{\begin{array}{l}
u_{D}=\Theta z_{D} \\
z_{D}=L \phi_{D}
\end{array}\right.
$$

where $\phi_{D}$ is a vector composed of the known basis functions for the solution of $u_{D}=\Theta z_{D}$, i.e., $\phi_{D}$ are the basis functions which make up the known form of the disturbance, and $L$ is a matrix of dimension ND by $\operatorname{dim}\left(\phi_{D}\right)$. The method for rejecting persistent disturbances used in this paper requires only the knowledge of the form of the disturbance, the amplitude of the disturbance does not need to be known, i.e. $(L, \Theta)$ can be unknown. In this paper, we will rejecting step disturbances of unknown amplitude which can be represented in the form of Eq. (3) as $\phi_{D} \equiv 1$, with $(L, \Theta)$ unknown.

In [7], as with much of the control literature, it is assumed that the plant and disturbance generator parameter matrices, $(A, B, C, \Gamma, \Theta, F)$, are known. This knowledge of the plant and its disturbance generator allows the Separation Principle of Linear Control Theory to be invoked to arrive at a State-Estimator based, linear controller that can suppress the persistent disturbances via feedback. In this paper, we will not assume that the plant and disturbance generator parameter matrices $(A, B, C, \Gamma, \Theta)$ are known. Instead, we assume that the disturbance generator parameter $F$ from Eq. (2) is known, i.e., the form of the disturbance functions is known. In many cases, knowledge of $F$ is not a severe restriction, since the disturbance function is often of known form but unknown amplitude.

Our control objective will be to cause the plant output $y_{p}(t)$ to asymptotically track the output of a known reference model $y_{m}(t)$. The reference model is given by:

$$
\left\{\begin{array}{l}
\dot{x}_{m}=A_{m} x_{m}+B_{m} u_{m} ; x_{m}(0)=x_{0}^{m} \\
y_{m}=C_{m} x_{m}
\end{array}\right.
$$

where the reference model state, $x_{m}(t)$, is an $\mathrm{N}_{\mathrm{m}}$-dimensional vector. The reference model output, $y_{m}(t)$, must have the same dimension as the plant output, $y_{p}(t)$. The excitation of the reference model is accomplished via the vector, $u_{m}(t)$, which is generated by:

$$
\dot{u}_{m}=F_{m} u_{m} ; u_{m}(0)=u_{0}^{m}
$$

It is assumed that the reference model is stable and the model parameters $\left(A_{m}, B_{m}, C_{m}, F_{m}\right)$ are known.

The desired control objective is for the output of the plant to asymptotically track the output of the reference model. We define the output error vector as:

$$
e_{y} \equiv y_{p}-y_{m}
$$


To achieve the desired control objective, we want $e_{y} \underset{t \rightarrow \infty}{\longrightarrow} 0$.

Consider the plant given by (1) with the disturbance generator given by (3). Our control objective for this system will be accomplished by an Adaptive Control Law of the form:

$$
u_{p}=G_{m} x_{m}+G_{u} u_{m}+G_{e} e_{y}+G_{D} \phi_{D}
$$

where $G_{m}, G_{u}, G_{e}$, and $G_{D}$ are matrices of the appropriate compatible dimensions whose definitions will be given later. Now we specify the adaptive gain laws that will produce asymptotic tracking:

$$
\left\{\begin{array}{l}
\dot{G}_{m}=-e_{y} x_{m}^{T} h_{m} \\
\dot{G}_{u}=-e_{y} u_{m}^{T} h_{u} \\
\dot{G}_{e}=-e_{y} e_{y}^{T} h_{e} \\
\dot{G}_{D}=-e_{y} \phi_{D}^{T} h_{D}
\end{array}\right.
$$

where $h_{m}, h_{u}, h_{e}$, and $h_{D}$ are arbitrary, positive definite matrices. The adaptive controller is specified by Eq. (8) with the adaptive gain laws given by Eq. (9). See [4] for an analysis of the stability of this controller and the proof of the following result:

Theorem 1: Suppose the following are true:

(1) All $u_{m}$ are bounded (i.e., all eigenvalues of $F_{m}$ are in the closed left-half plane);

(2) The reference model (4) is stable (i.e., all eigenvalues of $A_{m}$ are in the open left-half plane);

(3) $\phi_{D}$ is bounded (i.e., all eigenvalues of $F$ are in the closed left-half plane and any eigenvalues on the imaginary axis are simple);

(4) $(A, B, C)$ is Almost Strict Positive Real (ASPR), i.e., $C B>0$ and the open-loop transfer function is minimum phase.

Then the adaptive gains $G_{m}, G_{u}, G_{e}$, and $G_{D}$, remain bounded and asymptotic tracking occurs, i.e., $e_{y} \equiv y_{p}-y_{m} \underset{t \rightarrow \infty}{\longrightarrow} 0$.

\section{Residual Mode Filter Augmentation of Adaptive Controller}

In some cases the plant in Eq. (1) does not satisfy the ASPR requirement of the adaptive controller. Instead, there may be be a modal subsystem that inhibits this property. In [8]-[9] we developed theory to augment the adaptive controller using a Residual Mode Filter (RMF) to compensate for the troublesome modal subsystem, or the Q modes. Here we present new theory for augmented adaptive model reference controllers using RMF.

Let us assume that Eq. (1) can be partitioned into the following modal form:

$$
\left\{\begin{array}{l}
{\left[\begin{array}{c}
\dot{x} \\
\dot{x}_{Q}
\end{array}\right]=\left[\begin{array}{cc}
A & 0 \\
0 & A_{Q}
\end{array}\right]\left[\begin{array}{c}
x \\
x_{Q}
\end{array}\right]+\left[\begin{array}{c}
B \\
B_{Q}
\end{array}\right] u_{p}+\left[\begin{array}{c}
\Gamma \\
\varepsilon \Gamma_{Q}
\end{array}\right] u_{D}} \\
y_{p}=\left[\begin{array}{ll}
C & C_{Q}
\end{array}\right]\left[\begin{array}{c}
x \\
x_{Q}
\end{array}\right] ; \varepsilon \geq 0
\end{array}\right.
$$


Define $x_{p} \equiv\left[\begin{array}{c}x \\ x_{Q}\end{array}\right], A_{p}=\left[\begin{array}{cc}A & 0 \\ 0 & A_{Q}\end{array}\right], \quad B_{p}=\left[\begin{array}{c}B \\ B_{Q}\end{array}\right], \quad \Gamma_{p}=\left[\begin{array}{c}\Gamma \\ \varepsilon \Gamma_{Q}\end{array}\right], C_{p}^{T}=\left[\begin{array}{c}C \\ C_{Q}\end{array}\right]$, and the disturbance generator $\left\{\begin{array}{l}\dot{z}_{D}=F z_{D} \\ u_{D}=\theta z_{D}\end{array}\right.$. The output tracking error and control objective remain as in Eqs. (7)-(8), i.e., $e_{y} \equiv y_{p}-y_{m} \longrightarrow{ }_{t \rightarrow \infty} 0$. However, now we will only assume that the subsystem $(A, B, C)$ is Almost Strictly Positive Real, rather than the full un-partitioned plant $\left(A_{p}, B_{p}, C_{p}\right)$, and the modal subsystem $\left(A_{Q}, B_{Q}, C_{Q}\right)$ will be known and open-loop stable, i.e., $A_{Q}$ is stable. Also note that this subsystem is directly affected by the disturbance input. In [10] it was shown that a system $(A, B, C)$ is ASPR when $C B$ is positive definite and the open-loop system $P(s)=C(s I-A)^{-1} B$ is minimum phase. So, in summary, the actual plant has an ASPR subsystem and a known modal subsystem that is stable but inhibits the property of ASPR for the full plant. Hence, this modal subsystem must be compensated or filtered away.

We define the Residual Mode Filter (RMF):

$$
\left\{\begin{array}{l}
\dot{\hat{x}}_{Q}=A_{Q} \hat{x}_{Q}+B_{Q} u_{p} \\
\hat{y}_{Q}=C_{Q} \hat{x}_{Q}
\end{array}\right.
$$

And the compensated tracking error:

$$
\tilde{e}_{y} \equiv e_{y}-\hat{y}_{Q}
$$

Now let $e_{Q} \equiv \hat{x}_{Q}-x_{Q}$ and use substitution to obtain the error system:

$$
\dot{e}_{Q}=A_{Q} e_{Q}-\varepsilon \Gamma_{Q} u_{D}
$$

Consequently we can use Eq. (11) and the definition of $e_{Q}$ to write Eq. (12) as:

$$
\begin{aligned}
\tilde{e}_{y} & =\left(y_{p}-y_{m}\right)-C \hat{x}_{Q} \\
& =\left(C x+C_{Q} x_{Q}-C x_{m}\right)-\left(C_{Q} x_{Q}+C_{Q} e_{Q}\right) \\
& =C \Delta x-C_{Q} e_{Q}
\end{aligned}
$$

As in [1]-[2], we define the ideal trajectories $x_{*}$ and $u_{*}$ as a linear combination of the plant states, the control inputs, and the disturbance inputs. Note that this is done only for the ASPR subsystem $(A, B, C)$ as follows:

$$
\left\{\begin{array}{l}
\dot{x}_{*}=A x_{*}+B u_{*}+\Gamma u_{D} ; x_{*}(0)=x_{0} \\
y_{*}=C x_{*}=y_{m}
\end{array}\right.
$$

with $\left\{\begin{array}{l}x_{*}=S_{11}^{*} x_{m}+S_{12}^{*} u_{m}+S_{13}^{*} z_{D} \\ u_{*}=S_{21}^{*} x_{m}+S_{22}^{*} u_{m}+S_{23}^{*} z_{D}\end{array}\right.$.

Note that the ideal output $y_{*}$ matches the reference model output $y_{m}$. If such ideal trajectories exist, they will produce exact output tracking. The ideal trajectories can be made to match the reference model in Eqs. (4)-(5) with the following model matching conditions: 


$$
\left\{\begin{array}{l}
\bar{S}_{1}^{*} \bar{F}=A \bar{S}_{1}^{*}+B \bar{S}_{2}^{*}+\bar{\Gamma} \theta \\
C \bar{S}_{1}^{*}=\bar{C}_{m}
\end{array}\right.
$$

where $\bar{S}_{1}^{*} \equiv\left[\begin{array}{lll}S_{11}^{*} & S_{12}^{*} & S_{13}^{*}\end{array}\right\rfloor \quad \bar{S}_{2}^{*} \equiv\left\lfloor\begin{array}{lll}S_{21}^{*} & S_{22}^{*} & S_{23}^{*}\end{array}\right], \quad \bar{F} \equiv\left[\begin{array}{ccc}A_{m} & B_{m} & 0 \\ 0 & F_{m} & 0 \\ 0 & 0 & F\end{array}\right], \quad \bar{\Gamma} \equiv\left[\begin{array}{l}0 \\ 0 \\ \Gamma\end{array}\right], \quad$ and $\bar{C}_{m} \equiv\left[\begin{array}{lll}C_{m} & 0 & 0\end{array}\right]$

The model matching conditions given in Eq. (16) are necessary and sufficient conditions for the existence of ideal trajectories. Solutions to these matching conditions must exist for later analysis, but explicit solutions need never be known for the adaptive controller design. The model matching conditions are know to be uniquely solvable when $C B$ is nonsingular [10].

Let $\left\{\begin{array}{l}\Delta x \equiv x-x_{*} \\ \Delta u \equiv u_{p}-u_{*}, \text { then from Eqs. (14) and (15) we can write: } \\ \Delta \tilde{y} \equiv \tilde{e}_{y}\end{array}\right.$

$$
\left\{\begin{array}{l}
\Delta \dot{x}=A \Delta x+B \Delta u \\
\Delta \tilde{y}=C \Delta x-C_{Q} e_{Q}
\end{array}\right.
$$

This system can be rewritten as:

$$
\left\{\begin{aligned}
{\left[\begin{array}{l}
\Delta \dot{x} \\
\dot{e}_{Q}
\end{array}\right] } & =\left[\begin{array}{cc}
A & 0 \\
0 & A_{Q}
\end{array}\right]\left[\begin{array}{l}
\Delta x \\
e_{Q}
\end{array}\right]+\left[\begin{array}{l}
B \\
0
\end{array}\right] \Delta u+\left[\begin{array}{c}
0 \\
-\varepsilon \Gamma_{Q}
\end{array}\right] u_{D}=\bar{A}\left[\begin{array}{l}
\Delta x \\
e_{Q}
\end{array}\right]+\bar{B} \Delta u+\varepsilon \bar{\Gamma}_{Q} u_{D} \\
\Delta \tilde{y} & =\left[\begin{array}{ll}
C & -C_{Q}
\end{array}\right]\left[\begin{array}{l}
\Delta x \\
e_{Q}
\end{array}\right]=\bar{C}\left[\begin{array}{l}
\Delta x \\
e_{Q}
\end{array}\right]
\end{aligned}\right.
$$

Now we have the following:

Lemma: $\left(\bar{A}=\left[\begin{array}{cc}A & 0 \\ 0 & A_{Q}\end{array}\right], \bar{B}=\left[\begin{array}{l}B \\ 0\end{array}\right], \bar{C}=\left[\begin{array}{ll}C & -C_{Q}\end{array}\right]\right)$ is ASPR if and only if $(A, B, C)$ is ASPR.

Proof: $\bar{C} \bar{B}=\left[\begin{array}{ll}C & -C_{Q}\end{array}\right]\left[\begin{array}{l}B \\ 0\end{array}\right]=C B>0$ and

$$
\begin{aligned}
\bar{P}(s) & \equiv \bar{C}(s I-\bar{A})^{-1} \bar{B} \\
& =\left[\begin{array}{ll}
C & -C_{Q}
\end{array}\right]\left[\begin{array}{cc}
(s I-A)^{-1} & 0 \\
0 & \left(s I-A_{Q}\right)^{-1}
\end{array}\right]\left[\begin{array}{l}
B \\
0
\end{array}\right] \\
& =C(s I-A)^{-1} B=P(s)
\end{aligned}
$$

is minimum phase. End of proof.

So there exists $G_{e}^{*}$ such that $\left(\bar{A}_{C} \equiv \bar{A}+\bar{B} G_{e}^{*} \bar{C}, \bar{B}, \bar{C}\right)$ is Strictly Positive Real (SPR) when $(A, B, C)$ is ASPR. Consequently, as is well known from the Kalman-Yacubovic Theorem, there exists $\bar{P}, \bar{Q}>0$ such that 


$$
\left\{\begin{array}{l}
\bar{A}_{C}^{T} \bar{P}+\bar{P} \bar{A}_{C}=-\bar{Q} \\
\bar{P} \bar{B}=\bar{C}^{T}
\end{array}\right.
$$

We now write the augmented adaptive control law using RMF:

$$
\left\{\begin{array}{l}
u_{p} \equiv G_{m} x_{m}+G_{u} u_{m}+G_{e} \tilde{e}_{y}+G_{D} \phi_{D} \\
\tilde{e}_{y} \equiv y_{p}-\hat{y}_{Q} \\
\dot{\hat{x}}_{Q}=A_{Q} \hat{x}_{Q}+B_{Q} u_{p} \\
\hat{y}_{Q}=C_{Q} \hat{x}_{Q}
\end{array}\right.
$$

with adaptive gains given by:

$$
\left\{\begin{array}{l}
\dot{G}_{m}=-\tilde{e}_{y} x_{m} h_{m} ; h_{m}>0 \\
\dot{G}_{u}=-\tilde{e}_{y} u_{m} h_{u} ; h_{u}>0 \\
\dot{G}_{e}=-\tilde{e}_{y} \tilde{e}_{y}^{T} h_{e} ; h_{e}>0 \\
\dot{G}_{D}=-\tilde{e}_{y} \phi_{D}^{T} h_{D} ; h_{D}>0
\end{array}\right.
$$

Finally, we have the following stability result:

Theorem 3: In Eq. (10), let $(A, B, C)$ be ASPR, $A_{Q}$ be stable, and $\phi_{D}$ be bounded. Then the augmented adaptive controller using RMF in Eqs. (20)-(21) produces $e_{y}=y_{p}-y_{m}$ and $e_{Q}$ ultimately bounded into a ball of radius $R_{*} \equiv \varepsilon \frac{\left(1+\sqrt{p_{\max }}\right)}{a \sqrt{p_{\min }}} M_{v}$ with exponential rate and bounded adaptive gains $\left(G_{m}, G_{u}, G_{e}, G_{D}\right)$.

Proof: From Eq. (20), we have $u_{p} \equiv G_{m} x_{m}+G_{u} u_{m}+G_{e} \widetilde{e}_{y}+G_{D} \phi_{D}$, so we can write

$$
\begin{aligned}
& \Delta u \equiv u_{p}-u_{*} \\
& =\left[G_{m} x_{m}+G_{u} u_{m}+G_{e} \tilde{e}_{y}+G_{D} \phi_{D}\right]-\left[S_{21}^{*} x_{m}+S_{22}^{*} u_{m}+S_{23}^{*} L \phi_{D}\right] \\
& =G_{e}^{*} \tilde{e}_{y}+\Delta G \eta \\
& \text { where }\left\{\begin{array}{l}
\Delta G_{u} \equiv G_{u} \\
\Delta G_{m} \equiv G_{m} \\
\Delta G_{e} \equiv G_{e} \\
\Delta G_{D} \equiv G_{D} \\
\Delta G \equiv G- \\
\eta \equiv\left[\begin{array}{c}
x_{m} \\
u_{m} \\
\tilde{e}_{y} \\
\phi_{D}
\end{array}\right]
\end{array}\right.
\end{aligned}
$$


Then

$$
\left\{\begin{array}{l}
\dot{\zeta}=\bar{A} \zeta+\bar{B} \Delta u=\bar{A}_{C} \zeta+\bar{B} w+\varepsilon \bar{\Gamma}_{Q} u_{D} \\
\tilde{e}_{y}=\bar{C} \zeta
\end{array}\right.
$$

with $\zeta \equiv\left[\begin{array}{c}\Delta x \\ e_{Q}\end{array}\right], w \equiv \Delta G \eta, \bar{A}_{C} \equiv \bar{A}+\bar{B} G_{e}^{*} \bar{C}$.

From Eq. (21), we can see that

$$
\dot{G}=\Delta \dot{G}=-\widetilde{e}_{y} \eta^{T} h ; h \equiv\left[\begin{array}{cccc}
h_{m} & 0 & 0 & 0 \\
0 & h_{u} & 0 & 0 \\
0 & 0 & h_{e} & 0 \\
0 & 0 & 0 & h_{D}
\end{array}\right]>0
$$

Since $(A, B, C)$ is ASPR, and by the lemma, so is $(\bar{A}, \bar{B}, \bar{C})$, we can use the following result from [11] where $v \equiv \bar{\Gamma}_{Q} u_{D}$ is bounded because the disturbance $u_{D}=L \phi_{D}$ is bounded.

Result: Consider the nonlinear, coupled system of differential equations,

$$
\left\{\begin{array}{l}
\dot{\varsigma}=\bar{A}_{c} \varsigma+\bar{B}\left(G(t)-G^{*}\right) \eta+\varepsilon v \\
\tilde{e}_{y}=\bar{C} \varsigma \\
\dot{G}(t)=-\tilde{e}_{y} \eta^{\mathrm{T}} h-a G(t)
\end{array}\right.
$$

where $G^{*}$ is any constant matrix and $\mathrm{h}$ is any positive definite constant matrix, each of appropriate dimension. Assume the following:

i) the triple $(\bar{A}, \bar{B}, \bar{C})$ is $S P R$,

ii) there exists $M_{K}>0$ such that $\left\|\left(G^{*}\right)^{T} G^{*}\right\| \leq M_{K}$, using the trace norm,

iii) there exists $M_{v}>0$ such that $\sup _{t \geq 0}\|v(t)\| \leq M_{v}$,

iv) there exists $a>0$ such that $a \leq \frac{q_{\min }}{2 p_{\max }}$, and

v) $h$ satisfies $\left\|h^{-1}\right\|_{2} \leq\left(\frac{\varepsilon M_{V}}{a M_{K}}\right)^{2}$, where $p_{\min }, p_{\max }$ are the minimum and maximum eigenvalues of $\bar{P}$ and $q_{\min }$ is the minimum eigenvalue of $\bar{Q}$ in the system $\left\{\begin{array}{l}\bar{A}_{C}^{T} \bar{P}+\bar{P} \bar{A}_{C}=-\bar{Q} \\ \bar{P} \bar{B}=\bar{C}^{T}\end{array}\right.$.

Then the matrix $G(t)$ is bounded and the state $\zeta(t)$ exponentially approaches the ball of radius $R_{*} \equiv \varepsilon \frac{\left(1+\sqrt{p_{\max }}\right)}{a \sqrt{p_{\text {min }}}} M_{v}$ with $\varepsilon>0$. 
From this result, we have $\zeta$ is ultimately bounded into the ball of radius $R_{*}$ which leads to $e_{y} \equiv y_{p}-y_{m}=y_{p}-y_{*}=C \Delta$ and $e_{Q}$ ultimately bounded as well. Therefore $G=G_{*}+\Delta G$ is bounded, as desired. End of proof.

Consequently, the radius of the error ball $R_{*} \equiv \varepsilon \frac{\left(1+\sqrt{p_{\max }}\right)}{a \sqrt{p_{\min }}} M_{v}$ is determined by the size of $\varepsilon$, which is related to the amount of disturbance leakage into the $\mathrm{Q}$ modes. It can be seen that, when there is no leakage of the disturbance into the $\mathrm{Q}$ modes $(\varepsilon=0)$, the convergence is asymptotic to zero.

\section{Design of Wind Turbine Controller}

\section{A. Wind turbine simulation}

Rated wind speed is the velocity at which maximum power output, or rated power, of a wind turbine is achieved. If a turbine is allowed to operate in an uncontrolled manner, in conditions where the wind speed is above the rated wind speed, the power output would increase in proportion to the cube of the wind speed, resulting in overheating of the generator and the power electronics system. Additionally, high wind speeds result in larger aerodynamic forces on the machine, causing fatigue and possible system failure. Hence, power output of a turbine must be held constant for wind speeds at and above the turbine's rated wind speed. This wind turbine operation area is called Region 3 [12]. For variable-speed turbines operating in Region 3, a constant torque is applied at the generator, and the turbine rotational speed is maintained at the desired value through the use of collective blade pitch.

Wind turbine control problems can benefit from adaptive control, which are well suited to nonlinear applications that have unknown modeling parameters and poorly known operating conditions. The main nonlinearities in a wind turbine model come from the nonlinear aerodynamic loads on the turbine. Creating an accurate model of the dynamic characteristics of a wind turbine is expensive and extremely difficult, if not impossible. Additionally, wind turbines operate in highly turbulent and unpredictable conditions. These complex aspects of wind turbines make them attractive candidates for the application of adaptive control methods.

The Controls Advanced Research Turbine (CART2) is a two-bladed, upwind, active-yaw, variable-speed horizontal-axis wind turbine located at the National Wind Technology Center (NWTC) in Golden, Colorado. This machine is used as a test bed to study aspects of wind turbine control technology for medium-scale machines [1]. The pitch system on the CART uses electromechanical servos that can pitch the blades up to \pm 18 deg/s. In Region 3 , the CART uses a conventional variable-speed approach to maintain rated electrical power, which is $600 \mathrm{~kW}$ at a low-speed shaft [LSS] speed of 41.7 RPM and a high-speed shaft [HSS] speed of 1800 RPM. Power electronics are used to command constant torque from the generator and full-span blade pitch controls the turbine speed.

The CART has been modeled with the Fatigue, Aerodynamics, Structures, and Turbulence Codes (FAST) as a combination of rigid and flexible bodies connected by several degrees of freedom (DOFs). The DOFs can be turned on or off individually for analysis purposes by setting a switch in the input data file. The FAST Code is a comprehensive aeroelastic simulator capable of predicting both the extreme loads and the fatigue loads of two- and three-bladed horizontal axis wind turbines [13]. FAST uses Kane's method to set up equations of motion that are solved by numerical integration. The aerodynamic forces and moments along the turbine blade are calculated in FAST by the AeroDyn subroutine package [14].

\section{B. Region 3 Collective Pitch Controller}

In this section, we use the theory from section II to design region 3 controllers for the FAST simulation of the CART2. The parametric information for the FAST simulator as we configured it is available from [15]. For the adaptive controller with a reference model, the control objective is to track the model, to reject wind disturbances, and to operate in the presence of flexible modes using collective blade pitch. The model is designed such that output tracking results in plant generator speed regulation at $1800 \mathrm{rpm}$. An adaptive controller without a reference model as described in ref. [9] has the objective of regulating the generator speed instead of tracking a model. The inputs to the FAST plant are generator torque, blade pitch angle, and nacelle yaw. The FAST simulator can be configured to output many different states or measurements of the plant, such as generator speed and low speed shaft velocity. In this study, the yaw is assumed fixed, so that the major component of the wind inflow is normal to the rotor. In addition, the generator torque is assumed constant in region 3. Collective blade pitch is the only controller output. Turbine rotational speed, measured on the low-speed shaft side of the gearbox, is the only plant output used by the region 3 controllers. A classical PI collective pitch controller (the baseline PI pitch controller) has been implemented 
and tested in the FAST simulator and a similar controller was field tested on the CART2 [16]-[17] for validation of the codes. We use the baseline PI pitch controller as a basis for comparison with the adaptive pitch controller.

The adaptive collective pitch controllers designed for this paper replace the baseline PI pitch controller in the FAST simulator. The uniform wind disturbance, without shear, across the rotor disk of a turbine can be modeled as a step disturbance [18]. Hence, to improve controller performance and reduce loads due to changes in wind speed, we design the adaptive collective pitch controller to reject step disturbances of unknown amplitude. The control objectives are accomplished by collective blade pitch.

A control law of the form given in Eq. (21) with gains specified by Eq. (20) is used to design the model reference adaptive collective pitch controller. A step function is used as the disturbance generator function, i.e., $\phi_{D}=1$ from Eq. (2). Recall that the amplitude of the disturbance function does not need to be known. This adaptive controller is implemented in Simulink ${ }^{\mathrm{TM}}$ for the FAST simulation of the CART2. The reference model chosen was a linearized model including the generator state and the tower state. The poles corresponding to the tower first fore-aft bending mode were moved from $-0.0722 \pm 5.4999 i$ to $-0.2722 \pm 5.4999 i$ using output feedback. The adaptive controllers' gains were tuned to track the reference model, minimize the generator speed error, and reject the wind disturbances. The gains used in the model reference adaptive controller are: $h_{u}=0.01, h_{m}=0.1, h_{e}=8.5$ and $h_{D}=0.3$. The gains used in the regulating adaptive controller are:, $h_{e}=6.5$ and $h_{D}=0.3$.

In a previous study ${ }^{8}$, the blade first flap-wise bending DOF when enabled along with the drive-train mode, were shown to cause the adaptively controlled turbine to saturate the blade pitch actuators after 60 seconds of steady uniform wind inflow at $18 \mathrm{mps}$, causing the turbine to enter over-speed condition. The baseline PI controller remained within acceptable operating limits, but the generator speed tracking was unacceptable at wind speeds above $17 \mathrm{mps}$.

A stability condition of the adaptive controller is an ASPR plant, i.e., $C B$ positive definite and the open-loop transfer function of the plant has no non-minimum phase zeros. When the turbine simulation is trimmed at a wind speed of $18 \mathrm{mps}$ with the generator and drive-train DOFs enabled to obtain a linear model of the plant, the open-loop transfer function of the linearized plant model has two non-minimum phase zeros at $0.0111 \pm 5.499 i$. Hence the plant does not satisfy the ASPR condition for the adaptive controller. We use the theory from section II above to design a Residual Mode Filter to remove the non-minimum phase modes from the plant to restore the ASPR property of the plant.

A Residual Mode Filter is designed from the linear model by first converting the linear system to a modal system. We partition the modal system into two subsystems, one minimum phase subsystem and a second stable subsystem with two non-minimum phase zeros. The second subsystem contains the Q-modes, so it is used as the Residual Mode Filter given in Eq. (11) to augment the adaptive controller to remove the plant's non-minimum phase modes. The transfer function for the RMF is

$$
T(s)=\frac{790.52 s-38.32}{s^{2}+0.02 s+430.30}
$$

The RMF is placed in a loop around the controller in the Simulink ${ }^{\mathrm{TM}}$ model of the turbine. The controller output is fed to both the plant and the RMF. The RMF output is subtracted from the plant output before it is passed to the controller. The RMF removes the modes from the plant output that inhibit the ASPR property. Previous results from [14] showed that fixed gain controllers could be augmented with RMFs, so the same RMF is added to the original baseline PI pitch controller. An advantage of RMF augmentation is that it requires no modification to the control laws or control gains. The RMF is designed to remove only the Q-modes, whereas a low-pass filter attenuates or removes frequencies above a certain value. Next we present simulation results using these controllers.

\section{Simulation Results}

The baseline and model reference adaptive controllers augmented using the RMF with transfer function given by Eq. (22) were compared in simulation. Simulations were run with turbulent wind inflow to excite the nonlinear turbine model. The turbulent wind primarily results in region 3 operation, see fig. 1 . All of the degrees of freedom for the simulation were enabled. The resulting generator speed from the simulations are shown in fig. 2. The PI controller exceeds the generator speed limit at 30 seconds. Figure 3 shows the comparison of the model reference adaptive controller with the adaptive controller with no model (the regulating adaptive controller). Both adaptive controllers have comparable results. Preliminary studies of the tower bending modes did not show significant changes when the model was used with the adaptive controller. 




Fig. 1. Turbulent wind inflow used in simulations.

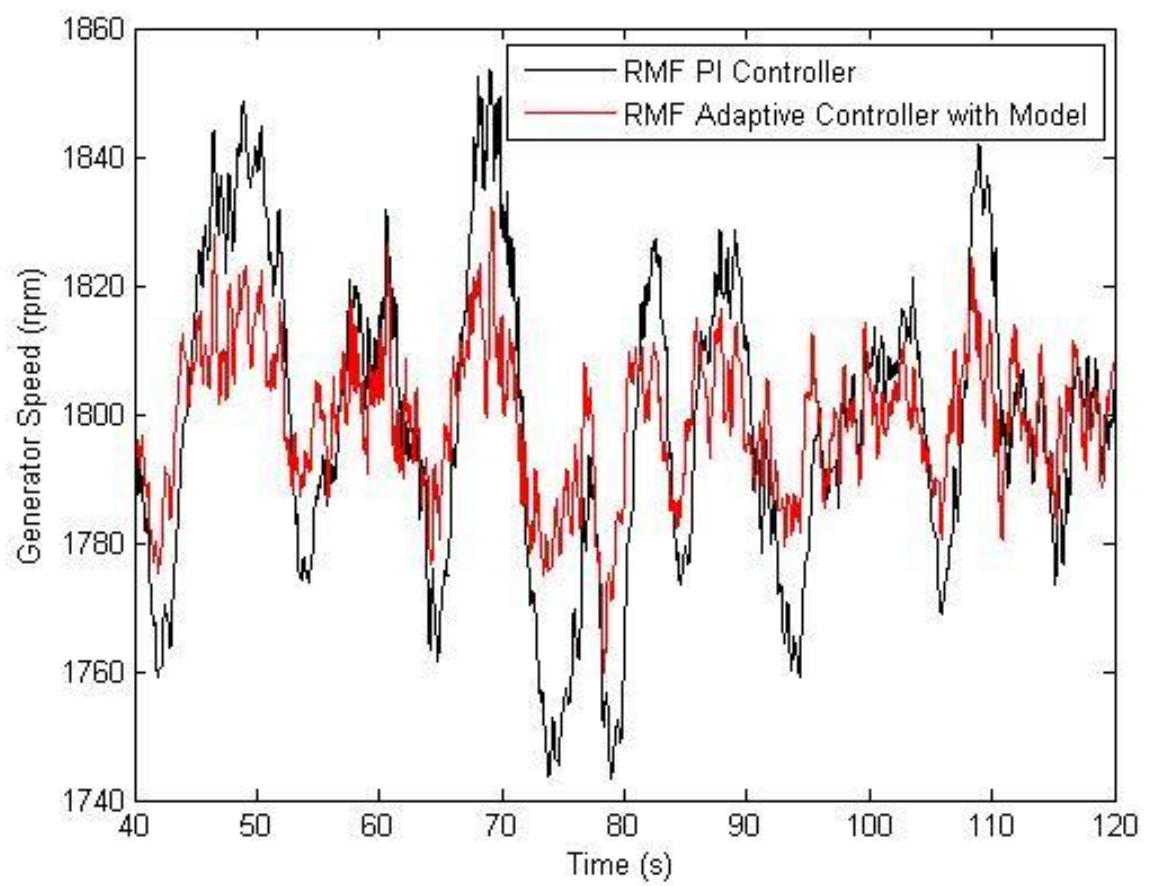

Fig. 2. Baseline PI with RMF and Reference Model Adaptive RMF with turbulent wind inflow. 


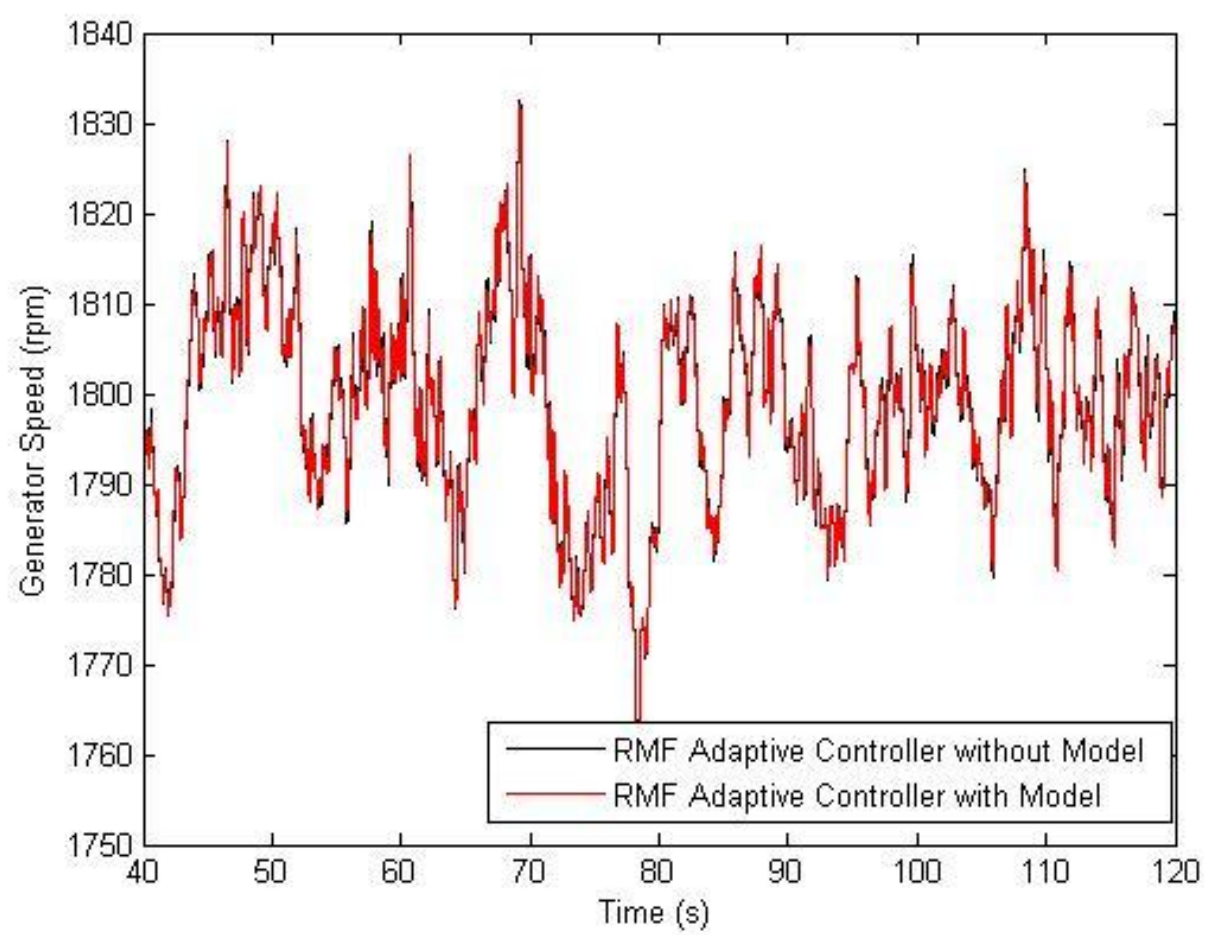

Fig. 3. Generator speed for RMF adaptive controllers with and without a reference model.

\section{Conclusion}

We have proposed an augmented model reference adaptive controller with adaptive rejection of persistent disturbances using a residual mode filter. The RMF is used to accommodate problematic modes in the system that inhibit the adaptive controller, in particular the ASPR condition. This new theory accounts for adaptive model tracking. This theory is used to design a controller for a utility scale wind turbine that outperformed the baseline PI controller. Future work will include investigating different turbine reference models to determine if they can have a positive affect on the loads experienced by the turbine.

\section{Acknowledgements}

The authors would like to thank the Subsonic Rotary Wing Project of the Fundamental Aeronautics Program of NASA, and in particular Ms. Susan Gorton and Dr. Colin Theodore for their support of this research.

\section{References}

${ }^{1}$ Wen, JT and Balas, MJ, "Robust adaptive control in Hilbert space," Journal of Mathematical Analysis and Application 1989; 143(1): 1-26.

${ }^{2}$ Balas, MJ, "Finite-dimensional direct adaptive control for discrete-time infinite-dimensional linear systems," Journal of Mathematical Analysis and Applications 1995; 196(1): 153-171.

${ }^{3}$ Fuentes, RJ, Balas, MJ, "Direct adaptive rejection of persistent disturbances," Journal of Mathematical Analysis and Applications 2000; 251(1): 28-39.

${ }^{4}$ Frost, SA, Balas, MJ, and Wright, AD, "Direct adaptive control of a utility-scale wind turbine for speed regulation," International Journal of Robust and Nonlinear Control, 2009, 19(1): 59-71, DOI: 10.1002/rnc.1329.

${ }^{5}$ Balas, MJ, Finite-dimensional controllers for linear distributed parameter systems: Exponential stability using Residual Mode Filters," J. Mathematical Analysis \& Applications, Vol. 133, pp. 283-296, 1988

${ }^{6}$ Johnson, C.D. Theory of disturbance-accommodating controllers. Control \& Dynamic Systems, Advances in Theory and Applications, Leondes, CT. ed. Academic Press: New York, 1976; 12: 387-489.

${ }^{7}$ Kimura, H. Pole assignment by gain output feedback. IEEE Trans. Automatic Control 1975; AC-20(4): 509-516.

${ }^{8}$ Frost, SA, Balas, MJ, and Wright, AD, "Modified adaptive control for region 3 operation in the presence of wind turbine structural modes," Proceedings 49th AIAA Aerospace Sciences Meeting, Orlando, 2010.

${ }^{9}$ Frost, SA, Balas, MJ, and Wright, AD, "Generator Speed Regulation in the Presence of Structural Modes through Adaptive Control using Residual Mode Filters”, to appear in Mechatronics, DOI: 10.1016/j.mechatronics.2011.01.006.

${ }^{10}$ Balas, M.J., Fuentes, R.J.; A Non-orthogonal projection approach to characterization of Almost Positive Real Systems with an application to adaptive control. Proceedings of the American Control Conference, Boston, 2004 
${ }^{11}$ Fuentes, R J and Balas, M J, "Robust Model Reference Adaptive Control with Disturbance Rejection", Proceedings of the American Control Conference, 2002.

${ }^{12}$ Fingersh, LJ, Johnson, KE. Baseline results and future plans for the NREL Controls Advance Research Turbine. Proceedings of the 23rd AIAA Aerospace Sciences Meeting and Exhibit Wind Energy Symposium 2004; 87-93.

${ }^{13}$ Jonkman, JM, Buhl, ML. FAST user's guide, National Renewable Energy Laboratory, NREL/EL-500-38230, Golden, Colorado, August, 2005.

${ }^{14}$ Laino, DJ, Hansen, AC. User's guide to the computer software routines AeroDyn interface for ADAMS®. Salt Lake City, Utah: Windward Engineering, LC, September 2001.

${ }^{15}$ National Renewable Energy Lab., NWTC Design Codes (FAST), http://wind.nrel.gov/designcodes/simulators/fast/, National Renewable Energy Lab., Golden, CO, March, 2010.

${ }^{16}$ Wright, A.D., Fingersh, L.J.; Advanced control design for wind turbines. Part I: Control design, implementation and initial tests. National Renewable Energy Laboratory, NREL/TP-500-42437, Golden, Colorado, March 2008.

${ }^{17}$ Wright, AD, Balas, MJ, Fingersh, LJ. Testing state-space controls for the controls advanced research turbine. Transactions of the ASME. Journal of Solar Energy Engineering 2006; 128(4): 506-515.

${ }^{18}$ Wright, AD, Balas, MJ. Design of controls to attenuate loads in the controls advanced research turbine. Transactions of the ASME. Journal of Solar Energy Engineering 2004; 126(4): 1083-9. 